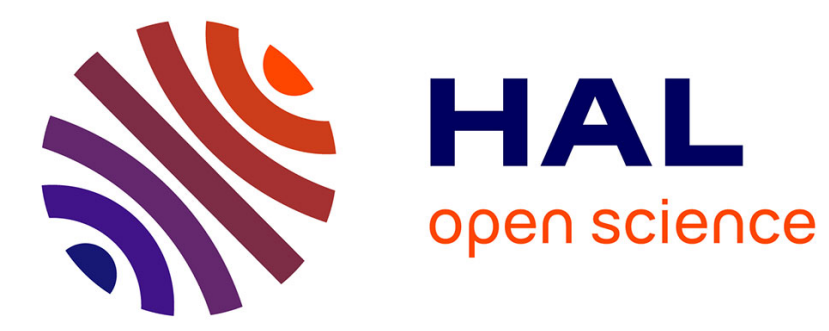

\title{
Submicron-diameter semiconductor pillar microcavities with very high quality factors
}

Guillaume Lecamp, Jean-Paul Hugonin, Philippe Lalanne, Rémy Braive, Spyros Varoutis, Sabine Laurent, Aristide Lemaître, Isabelle Sagnes, Gilles Patriarche, Isabelle Robert-Philip, et al.

\section{To cite this version:}

Guillaume Lecamp, Jean-Paul Hugonin, Philippe Lalanne, Rémy Braive, Spyros Varoutis, et al.. Submicron-diameter semiconductor pillar microcavities with very high quality factors. Applied Physics Letters, 2007, 90 (9), pp.091120. 10.1063/1.2711186 . hal-00877855

\section{HAL Id: hal-00877855 \\ https://hal-iogs.archives-ouvertes.fr/hal-00877855}

Submitted on 29 Oct 2013

HAL is a multi-disciplinary open access archive for the deposit and dissemination of scientific research documents, whether they are published or not. The documents may come from teaching and research institutions in France or abroad, or from public or private research centers.
L'archive ouverte pluridisciplinaire HAL, est destinée au dépôt et à la diffusion de documents scientifiques de niveau recherche, publiés ou non, émanant des établissements d'enseignement et de recherche français ou étrangers, des laboratoires publics ou privés. 


\title{
Submicron-diameter semiconductor pillar microcavities with very high quality factors
}

\author{
G. Lecamp, J. P. Hugonin, and P. Lalanne ${ }^{a)}$ \\ Laboratoire Charles Fabry de I'Institut d'Optique, CNRS, Univ. Paris-Sud, Campus Polytechnique, \\ RD 128, 91127 Palaiseau, France \\ R. Braive, S. Varoutsis, S. Laurent, A. Lemaître, I. Sagnes, G. Patriarche, \\ I. Robert-Philip, and I. Abram \\ Laboratoire de Photonique et de Nanostructures/CNRS, Route de Nozay, 91460 Marcoussis, France
}

(Received 27 October 2006; accepted 30 January 2007; published online 2 March 2007)

\begin{abstract}
Pillar microcavities are subject to two common fabrication artifacts: Bragg mirror corrugation and oxide deposit cladding. In this letter the authors investigate the impact of these features on the quality factor. A quasiperiodic variation of the quality factor as a function of the pillar diameter is experimentally observed and well described by theory. Moreover, observation of quality factors in excess of 1500 , close to the theoretical limit, is reported for 600-nm-diameter GaAs micropillars bounded by AlGaAs/GaAs Bragg mirrors. (C) 2007 American Institute of Physics.
\end{abstract}

[DOI: $10.1063 / 1.2711186]$

Pillar microcavities constitute an important class of optical devices in which light is strongly confined in all three dimensions of space, through Bragg reflection in the longitudinal direction and through total internal reflection in the transverse directions. The main advantage of micropillars compared with other types of microcavities (such as photonic crystal cavities) is that light escapes very efficiently in the direction parallel to the pillar axis in a single-lobe Gaussian-like pattern. For this reason, such cavities are well suited for a wide range of applications, requiring good light extraction, such as directional emitters or efficient single photon sources. ${ }^{1-3}$ At the same time, these resonators combine a small cavity volume $V$ and a relatively high quality factor $Q$, a feature that opens the way to the implementation of cavity quantum electrodynamics (CQED) effects in the solid state. Two such examples are (1) the Purcell effect, ${ }^{4,5}$ whose strength depends on the ratio $Q / V$ and has been used for the generation of indistinguishable single photons, ${ }^{6-8}$ and (2) the strong coupling regime between a single quantum dot and the cavity field, whose strength depends on the ratio $Q^{2} / V$ and has been recently observed in few-micron diameter micropillars. ${ }^{9}$ In view of the key role played by the quality factor and the modal volume in micropillar cavities for CQED effects, it is important to understand the physical and technical limitations of $Q$ in these cavities with small $V$, namely, in the submicron diameter range.

In this letter, we present a systematic theoretical and experimental study of the quality factor of submicron pillars $(d<1.5 \mu \mathrm{m})$. In this diameter range, it was generally admitted $^{10}$ that a drastic decrease of $Q$ was inevitable because of the fabrication process by reactive ion etching ${ }^{11}$ that induces fabrication artifacts. The two main noticeable artifacts (see Fig. 1) are the following: (1) the GaAs/AlGaAs Bragg mirrors, surrounding the cavity spacer, are corrugated because of partial oxidation of the AlGaAs layer when in contact with air, leading to a friable oxide and (2) the pillars are sheathed by a thin nonstochiometric silica $\left(\mathrm{SiO}_{x}\right)$ deposit. The results presented in this letter show that the influence of

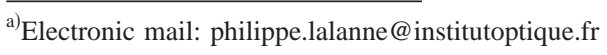

these fabrication features on the cavity $Q$ is not a simple decrease but is more complex and interesting. In order to obtain an in-depth understanding of their impact on the cavity performance, we first carry out three-dimensional (3D) fully vectorial calculations for realistic micropillars geometries, including corrugated mirrors and oxide cladding. Then we measure the quality factors of a series of micropillars with different subwavelength diameters and compare the experimental values with the theoretical results. For pillar diameters greater than $700 \mathrm{~nm}$ we observe, both theoretically and experimentally, specific oscillations of $Q$ as a function of the pillar diameter (see Fig. 3). The good agreement between the theoretical and experimental results in the variation of $Q$ as a function of the pillar diameter indicates that the two artifacts examined in this letter are the main factors influencing $Q$. For even smaller diameters, for which the corrugation depth and the oxide thickness correspond to a significant fraction of the diameter, agreement is not as good, indicating that additional factors, such as the nonuniformity of the corrugation and the oxide layer, must be taken into account to improve the model.
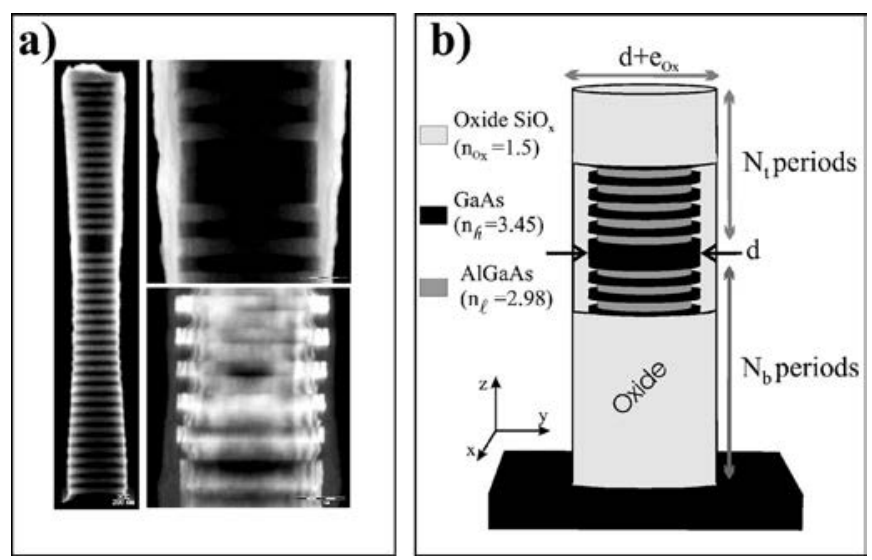

FIG. 1. Micropillar geometry used in this work. (a) Cross-sectional TEM 002 dark-field image of a 570-nm-diameter micropillar (left and top-right figures: 002 dark-field images and bottom-right figure: TEM 111 dark-field image). (b) Schematic view of a micropillar showing the parameters used in the theoretical computations. 

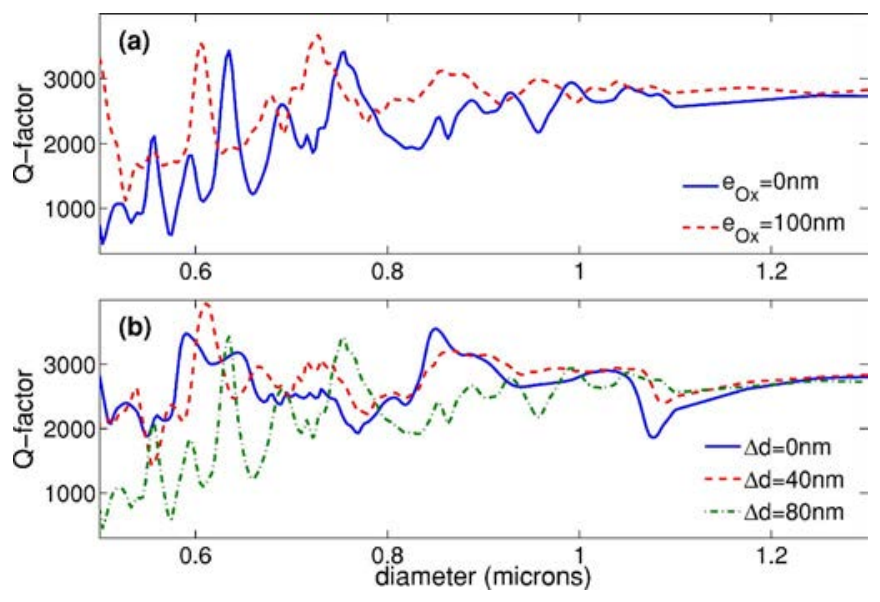

FIG. 2. (Color online) Numerical study of the impact of the oxide-adlayer cladding and of the DBR corrugation $\Delta d$ on the micropillar $Q$. (a) Impact of the oxide adlayer. $Q$ factors as a function of pillar diameter for oxide thickness $e_{\mathrm{ox}}=0 \mathrm{~nm}$ (solid blue curve) and $100 \mathrm{~nm}$ (dashed red curve). Calculations are performed with corrugation $(\Delta d=80 \mathrm{~nm}$ ). (b) Impact of the corrugation depth. $Q$ factors as a function of pillar diameter for $e_{\mathrm{ox}}=0 \mathrm{~nm}$ and for several corrugation depths $\Delta d=0 \mathrm{~nm}$ (solid blue curve), $\Delta d=40 \mathrm{~nm}$ (dashed red curve), and $\Delta d=80 \mathrm{~nm}$ (dashed-dotted green curve).

All pillars used in this study were fabricated from the same planar microcavity sample, grown by molecular beam epitaxy. The microcavities are composed of a $274 \mathrm{~nm} \mathrm{GaAs}$ spacer, surrounded by $N_{t}=15$ and $N_{b}=23$ periods of alternating $\mathrm{Al}_{0.9} \mathrm{Ga}_{0.1} \mathrm{As} / \mathrm{GaAs}$ layers, forming two distributed Bragg reflectors (DBRs). Every pair of layers consists of a 68-nm-thick GaAs layer and a 80-nm-thick $\mathrm{Al}_{0.9} \mathrm{Ga}_{0.1} \mathrm{As}$ layer. The optically active medium is composed of three layers of InAs self-assembled quantum dots grown in the middle of the GaAs spacer with a density of approximately $4 \times 10^{10} \mathrm{~cm}^{-2}$. Arrays of different pillars with diameters ranging from $550 \mathrm{~nm}$ to $1.3 \mu \mathrm{m}$ were fabricated through a combination of electron-beam lithography and $\mathrm{SiCl}_{4} / \mathrm{O}_{2}$ dry etching. ${ }^{11} \mathrm{~A}$ cross-sectional transmission electronic microscopy (TEM) image of a 570-nm-diameter micropillar is shown in Fig. 1(a). This type of image has been used for accurately measuring the different layer thicknesses. It shows that (1) the sidewalls are not straight but are corrugated with a $80 \mathrm{~nm}$ mean reduction of the $\mathrm{Al}_{0.9} \mathrm{Ga}_{0.1} \mathrm{As}$ layer diameter, compared with the GaAs layer diameter $d$, and that (2) they are clad with a $\mathrm{SiO}_{x}$ adlayer deposit whose thickness $e_{\mathrm{ox}}$ varies from $170 \mathrm{~nm}$ at the top of the pillars to $0 \mathrm{~nm}$ at the bottom and is around $100 \mathrm{~nm}$ in the vicinity of the cavity spacer. This adlayer is essential for the durability of the resonator since it prevents the formation of a friable aluminum oxide through the spontaneous oxidation of the $\mathrm{Al}_{0.9} \mathrm{Ga}_{0.1} \mathrm{As}$ layers when in contact with air.

In order to assess the impact of the oxide cladding and of the corrugation on the quality factor $Q$ of the cavity mode, we performed $3 \mathrm{D}$ fully vectorial calculations, using a modal approach that, in the transverse directions, relies on a Fourier expansion technique and assumes perfectly matched layers, while it uses analytical integration along the longitudinal pillar direction. ${ }^{12}$ Figure 2 summarizes the main results for 15-23 period DBR microcavities and for several corrugation depths, $\Delta d=0,40$, and $80 \mathrm{~nm}$, and two oxide thicknesses, $e_{\mathrm{ox}}=0$ and $100 \mathrm{~nm}$. The other geometrical and physical parameters used in the calculation are defined in Fig. 1(b). The first striking result that can be seen in Fig. 2 is a quasiperiDownloaded 17 Jun 2009 to 129.175.97.14. Redistribution subject odic variation of $Q$ as a function of the micropillar diameter. The physical origin of these variations, namely, the coupling of the fundamental $\mathrm{HE}_{11}$ guided mode of the spacer to a propagative Bloch mode in the DBR mirrors whose strength depends on the overlap between these two modes, has been explained for $\Delta d=e_{\mathrm{ox}}=0 \mathrm{~nm}$ in a previous publication ${ }^{13}$ and will not be further discussed here. We simply note that, as expected, the same mechanism holds for corrugated and oxide-clad micropillars.

More interesting, in the present context, is the fact that the presence of the oxide adlayer does not result in a $Q$ factor reduction but only in a displacement of the $Q$ variation pattern to smaller diameters, with a slight enhancement of $Q$ being even observed on Fig. 2(a) for $e_{\mathrm{ox}}=100 \mathrm{~nm}$. The impact of the corrugation depth on the cavity mode $Q$ factor is subtler. For moderate depths $(\Delta d=40 \mathrm{~nm})$ slightly smaller than that observed in the TEM picture of Fig. 1(a), the computed $Q$ values are similar to those of the uncorrugated case $(\Delta d=0 \mathrm{~nm})$ and for micropillars of approximately $600 \mathrm{~nm}$ diameter the $Q$ values exceed those of the planar cavity $\left(Q_{\mathrm{pl}} \approx 2800\right)$. By contrast, for larger corrugations $(\Delta d$ $=80 \mathrm{~nm}$ ), the cavity $Q$ 's rapidly decrease for $d<700 \mathrm{~nm}$. The physical origin of this trend is not obvious and deserves more theoretical studies, but we believe that it can be accounted for by the transverse mode-profile mismatch between the fundamental $\mathrm{HE}_{11}$ spacer guided mode and its associated evanescent DBR-Bloch modes: ${ }^{14}$ as the corrugation depth increases, the overlap between these two modes decreases and the net effect is a lowering of the Bragg mirror effective reflectivity, resulting in a decrease of the average $Q$ factor, as evidenced by Fig. 2(b) for small $d$ 's.

In order to confirm these numerical results and to estimate the impact of additional extrinsic loss mechanisms not taken into account in the numerical model (such as sidewall roughness, local variation of corrugation depth and oxide thickness as well as a residual small cladding absorption), the $Q$ 's of the processed pillars have been measured using the photoluminescence from the InAs quantum dots. The experimental data are obtained from microphotoluminescence measurements of single pillars in samples consisting of nine ideally identical pillars for 34 different diameters varying from $550 \mathrm{~nm}$ to $1.3 \mu \mathrm{m}$. The InAs quantum dots are excited nonresonantly by a continuous Ti:sapphire laser tuned to $810 \mathrm{~nm}$ and focused on the sample by a microscope objective (numerical aperture $=0.4$ ). The sample is kept at a temperature of $4 \mathrm{~K}$ and the luminescence, collected by the same objective, is sent to a $0.32 \mathrm{~m}$ spectrometer $(\approx 0.1 \mathrm{~nm}$ resolution) with a cooled silicon change coupled device. The quality factors of each pillars are deduced from the width of their photoluminescence spectra and the diameters of the pillars are deduced from the energy of the fundamental $\mathrm{HE}_{11}$ mode.

Figure 3 shows a comparison between the $Q$ factors measured on the sample and those calculated for realistic pillar geometries, with $e_{\mathrm{ox}}=100 \mathrm{~nm}$ and $\Delta d=80 \mathrm{~nm}$. The black curve superimposed on the experimental data in Fig. 3(a) is obtained by averaging over the different cavity mode energies in order to account for pillar-diameter dispersion due to technological inaccuracies. For large diameters, $d>1 \mu \mathrm{m}$, diameter fluctuations do not strongly impact the cavity mode energies so that for each series of nine ideally identical pillars, their cavity mode energies are very close. In this case, we can extract a mean $Q$ value for each series by simply averaging the measured $Q$ 's over the nine pillars of to AIP license or copyright; see http://apl.aip.org/apl/copyright.jsp 


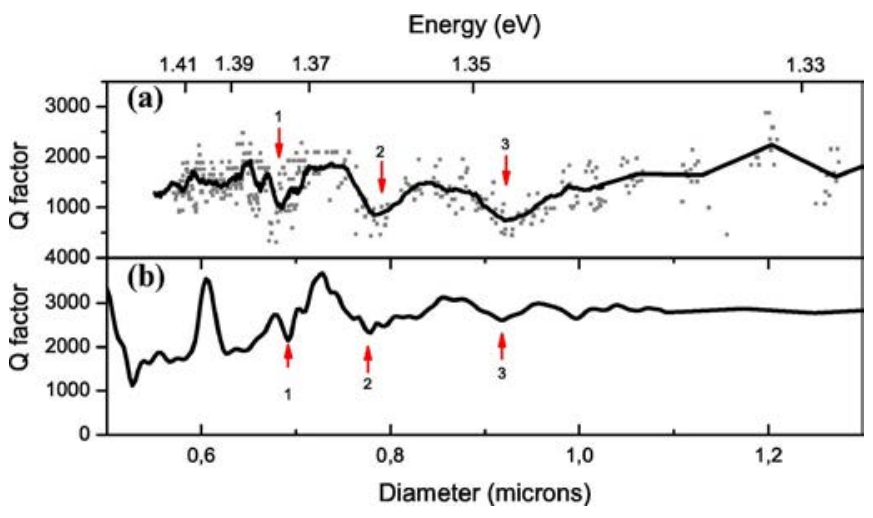

FIG. 3. (Color online) Comparison between (a) experimental and (b) computational results, displaying the pillar $Q$ factors as a function of the cavity diameter $d$ (lower scale) and of the energy (upper scale) of the fundamental cavity mode. The gray dots are the $Q$ factors measured for different pillars and the black curve is obtained by averaging over adjacent experimental data points. The computational data (b) are obtained for the geometric parameters actually measured on the sample with TEM pictures [see Fig. 1(a)], namely, for $\Delta d=80 \mathrm{~nm}$ and $e_{\mathrm{ox}}=100 \mathrm{~nm}$. The arrows indicate the position of $Q$ minima.

the series. However, for $d<1 \mu \mathrm{m}$, the cavity mode energies rapidly vary with $d$ so that the same mode energy can be measured for pillars belonging to different diameter series. For the sake of clarity, we have removed these fluctuations by averaging the measured $Q$ 's over a $N$-nearest-neighbor window. The black curve in Fig. 3(a) is obtained for $N=15$, but actually does not depend on $N$. Virtually identical results showing the same minima are obtained for $N=10$ and 20.

The discrepancies between the theoretical and experimental results can be easily understood: the fact that experimental $Q$ 's are systematically smaller than the theoretical values can be attributed to the optical quality of the cladding, in which there is probably a slight absorption. Then, the discrepancies observed for pillars with $d<700 \mathrm{~nm}$ and, in particular, the strong peak predicted by the model at $d=600 \mathrm{~nm}$, which is not observed experimentally, are probably due to the fact that for small diameters, the relative size of the corrugation and of the oxide thickness becomes critical, and the local variations of these parameters are no longer negligible. Clearly, at small diameters, a more complete model would be necessary to take into account the local variations, as observed on the TEM images. Nevertheless, a comparison of the experimental and theoretical figures shows good qualitative agreement for pillars with $d>700 \mathrm{~nm}$. In particular, the variation of the measured $Q$ 's as a function of $d$ exhibits oscillations, with minima at 690, 780, and $920 \mathrm{~nm}$ in agreement with the theoretical model and for $d=730 \mathrm{~nm}$ the experimental $Q$ values reach and almost rise above the $Q$ of the planar cavity.

These observations strongly support the theory in Ref. 13 and the computational model used in this work. It should also be noted that for the thinnest pillars measured $(d \approx 570 \mathrm{~nm})$, the $Q$ factor reaches a value of 1500 , only slightly smaller than the theoretical value of 1800 , underscoring the good quality of the fabrication process. Moreover, these cavities with diameters in the range $d$ $\approx 500-600 \mathrm{~nm}$ possess a mode volume of the order of $2(\lambda / n)^{3}$ and so potentially offer Purcell factors as high as 35 with very efficient light extraction.

In conclusion, by studying theoretically and experimentally the quality factor of micropillars with submicron diameters, displaying fabrication-induced corrugated mirrors, and oxide cladding, we have developed an accurate model that accounts for the diameter-dependent variations of $Q$, for micropillars with $d>700 \mathrm{~nm}$. We expect that this analysis will be helpful for further optimizations of the design and fabrication of micropillars. In particular, by tailoring the corrugation and the oxide thickness, it may be possible to fabricate micropillars with $Q$ 's larger than those reported here and also larger than the planar microcavity, provided that a given pillar diameter is targeted. Combined with a very efficient light extraction, such high- $Q$ and small- $V$ resonators are promising candidates for the implementation of quantum electrodynamics effects in the solid state.

This work was partly supported by the "Région Ile de France," the "Conseil Général de l'Essonne," and the French Ministry of Research under projects "Polqua" and "NanoQUB." The authors also acknowledge support from the "SANDiE" and "Epixnet" Networks of Excellence of the European Commission and would like also to thank A. Beveratos and J. M. Gérard for fruitful discussions.

${ }^{1}$ E. Moreau, I. Robert, J.-M. Gérard, I. Abram, L. Manin, and V. ThierryMieg, Appl. Phys. Lett. 79, 2865 (2001).

${ }^{2}$ W. L. Barnes, G. Björk, J. M. Gérard, P. Jonsson, J. Wasey, P. Worthing, and V. Zwiller, Eur. Phys. J. D 18, 197 (2002).

${ }^{3}$ M. Pelton, C. Santori, J. Vuckovic, B. Y. Zhang, G. S. Solomon, J. Plant, and Y. Yamamoto, Phys. Rev. Lett. 89, 233602 (2002).

${ }^{4}$ E. M. Purcell, Phys. Rev. 69, 681 (1946).

${ }^{5}$ J.-M. Gérard, B. Sermage, B. Gayral, B. Legrand, E. Costard, and V. Thierry-Mieg, Phys. Rev. Lett. 81, 1110 (1998).

${ }^{6}$ C. Santori, D. Fattal, J. Vuckovic, G. Solomon, and Y. Yamamoto, Nature (London) 419, 594 (2002).

${ }^{7}$ S. Varoutsis, S. Laurent, P. Kramper, A. Lemaître, I. Sagnes, I. RobertPhilip, and I. Abram, Phys. Rev. B 72, 041303 (2005).

${ }^{8}$ A. J. Bennett, D. C. Unitt, A. J. Shields, P. Atkinson, and D. A. Ritchie, Opt. Express 13, 7772 (2005).

${ }^{9}$ J. P. Reithmaier, G. Sek, A. Loffler, C. Hofmann, S. Kuhn, S. Reitzenstein, L. V. Keldysh, V. D. Kulakovskii, T. L. Reinecke, and A. Forchel, Nature (London) 432, 197 (2004).

${ }^{10}$ T. Rivera, J. P. Debray, J. M. Gérard, B. Legrand, L. Manin-Ferlazzo, and J. L. Oudar, Appl. Phys. Lett. 74, 911 (1999).

${ }^{11}$ S. Varoutsis, S. Laurent, I. Sagnes, A. Lemaître, L. Ferlazzo, C. Mériadec, G. Patriarche, I. Robert-Philip, and I. Abram, J. Vac. Sci. Technol. B 23, 2499 (2005).

${ }^{12}$ E. Silberstein, P. Lalanne, J. P. Hugonin, and Q. Cao, J. Opt. Soc. Am. A 18, 2865 (2001).

${ }^{13}$ P. Lalanne, J. P. Hugonin, and J. M. Gérard, Appl. Phys. Lett. 84, 4726 (2004).

${ }^{14}$ G. Lecamp, P. Lalanne, J. P. Hugonin, and J. M. Gérard, IEEE J. Quantum Electron. 41, 1323 (2005). 